\title{
Ganho compensatório em cordeiras na fase de recria: desempenho e medidas biométricas
}

\author{
Antonio Carlos Homem Junior ${ }^{1}$, Américo Garcia da Silva Sobrinho ${ }^{2}$, Sandra Mari Yamamoto 3 , \\ Rafael Silvio Bonilha Pinheiro ${ }^{4}$, Carolina Buzzulini ${ }^{4}$, Carolina Spina Alves de Lima ${ }^{5}$ \\ 1 Graduando em Zootecnia da FCAV/Unesp e bolsista PIBIC/CNPq/Unesp. Via de Acesso Prof. Paulo Donato Castellane, s/no. CEP: \\ 14884-900, Jaboticabal, SP. \\ 2 Departamento de Zootecnia da FCAV/Unesp, Jaboticabal, SP \\ ${ }^{3}$ Doutorando em Zootecnia (Produção Animal) da FCAV/Unesp, Jaboticabal, SP. \\ ${ }^{4}$ Mestrando em Zootecnia (Produção Animal) da FCAV/Unesp, Jaboticabal, SP. \\ 5 Graduando em Zootecnia da FCAV/Unesp, Jaboticabal, SP.
}

RESUMO - Objetivou-se estudar o ganho compensatório de cordeiras submetidas a restrição alimentar e, posteriormente, a realimentação à vontade. Foram adotados dois períodos de 60 dias, de modo que, no primeiro, 18 cordeiras $7 / 8$ Ile de France 1/8 Ideal foram distribuídas em três tratamentos, em delineamento inteiramente casualisado: sem restrição = alimentação à vontade por todo o experimento; restrição $30 \%=$ restrição alimentar de $30 \%$ em relação ao consumo do grupo sem restrição; e restrição $60 \%$ = restrição alimentar de $60 \%$ em relação ao consumo do grupo sem restrição. No segundo período, todas as cordeiras receberam alimentação à vontade. Ao final do primeiro período, as cordeiras alimentadas à vontade e aquelas sob restrição alimentar de $30 \%$, tiveram ganho de peso corporal de 18 e $0,8 \%$, respectivamente, enquanto aquelas sob restrição alimentar de $60 \%$ perderam $15 \%$ de peso corporal. No segundo período, todas as cordeiras ganharam peso, observando-se maior ganho naquelas sob restrição $30 \%$ (196,24 g/dia) em relação às sem restrição (116,20 g/dia). O ganho de peso desses dois grupos, no entanto, não diferiu do grupo restrição $60 \%(178,03 \mathrm{~g} / \mathrm{dia})$. A conversão alimentar das cordeiras alimentadas à vontade foi 10,09 e a daquelas com restrição alimentar de $30 \%$ foi de 5,97. As medidas biométricas foram semelhantes no início do experimento, mas, ao final da restrição alimentar, houve diminuição de $16 \%$ na largura do ombro, de $21 \%$ na largura da garupa, de $6,9 \%$ no perímetro torácico e de $39 \%$ na condição corporal das cordeiras do grupo restrição $60 \%$ em relação às medidas iniciais. A restrição alimentar de $60 \%$ resultou em menor consumo de MS e em peso corporal final mais baixo, ocasionando prejuízos na maioria das medidas corporais. A restrição alimentar de $30 \%$, no entanto, pode ser indicada como alternativa de manejo alimentar para melhorar a conversão alimentar e reduzir o consumo total de alimento.

Palavras-chave: ovinos, realimentação, restrição alimentar

\section{Compensatory body weight gain in growing lambs: performance and biometrical measurements}

\begin{abstract}
The objective of this trial was to evaluate the compensatory growth and biometrical measurements on lambs feed restricted or ad libitum fed. In the first of two periods of 60 days, 18 7/8 Ile de France x 1/8 Ideal female lambs were assigned to three treatments in a completely randomized design as follows: 1) no feed restriction (ad libitum fed), 2) $30 \%$ of feed restriction or 3) $60 \%$ of feed restriction calculated as proportion of the ad libitum fed group intake. In the second period, all animals were ad libitum fed. At the end of the first period, lambs with no feed restriction and those with $30 \%$ of feed restriction gained 18 and $0.8 \%$ of body weight $(\mathrm{BW})$, respectively, compared to their initial weight while lambs with $60 \%$ of feed restriction lost $15 \%$ of BW. In the second period, BW gain was greatest on animals with $30 \%$ of feed restriction $(196.24 \mathrm{~g} / \mathrm{d})$, lowest on those not feed restricted $(116.20 \mathrm{~g} / \mathrm{d})$ and intermediate on animals with $60 \%$ of feed restriction. Feed conversion was of 10.09 and of 5.97 on lambs not feed restricted and with $30 \%$ of feed restriction, respectively. The biometrical measurements were similar at the beginning of the trial but at the end of the first period there were reductions of $16 \%$ on shoulder width, $21 \%$ on hip width, $6.9 \%$ on chest girth, and $39 \%$ on body condition in animals with $60 \%$ of feed restriction. Feed restriction of $60 \%$ also reduced DM intake and final BW of lambs, which compromised most of the biometrical measurements. On the other hand, $30 \%$ of feed restriction can be an alternative feeding management because of the observed improvement in feed conversion and decreased total feed intake.
\end{abstract}

Key Words: feed restriction, refeeding, sheep 


\section{Introdução}

O Brasil é o 14으 produtor mundial de ovinos, com produção anual de mais de 14 milhões de cabeças (Anualpec, 2004). A demanda de carne ovina cresceu significativamente nos últimos anos, principalmente nos grandes centros urbanos, resultando em uma expansão da ovinocultura de corte em estados que antes não tinham tradição nesta atividade, como São Paulo, Espírito Santo, Minas Gerais, Paraná e Mato Grosso do Sul. A diversidade das condições ambientais destas áreas tem intensificado os sistemas de criação, destacando o desmame precoce, a recria e a terminação de cordeiros em regime de confinamento como alternativas para maximizar a produção ovina (Siqueira, 1996).

Para o sucesso destes sistemas, onde a alimentação é um dos fatores que mais oneram o custo de produção, é imprescindível o aprofundamento no segmento nutricional, haja vista sua estreita relação com o retorno econômico da produção de cordeiros, determinando as interações níveis nutricionais $\times$ respostas fisiológicas que modificam a composição corporal e a conversão alimentar com a finalidade de aproveitar toda a potencialidade produtiva dos animais a um custo de produção adequado (Gerassev et al., 2006).

O manejo nutricional do nascimento ao primeiro parto influencia o potencial reprodutivo da ovelha. Uma nutrição inadequada dos animais de reposição reduz as eficiências produtiva e reprodutiva do rebanho, resultando em menor vida útil da fêmea e em menor pressão de seleção. Desde que não haja deposição excessiva de gordura, a adequada alimentação na fase de recria permite que borregas sejam acasaladas mais cedo, ao atingirem $70 \%$ do peso adulto, com primeiro parto aos 12 a 14 meses de idade (Susin, 1996).

Quando a quantidade de nutrientes oferecida aos animais é restrita, as fases de crescimento normal do animal são comprometidas. O crescimento pós-natal pode ser dividido em quatro fases, a primeira caracterizada pelo rápido crescimento de cabeça, pescoço e patas; a segunda, pelas mudanças na conformação corporal; a terceira, dos 3 aos 4 meses, pelo aumento generalizado do organismo, iniciando a deposição de gordura; e a quarta, pela concentração do crescimento nas massas musculares das extremidades e do lombo e pelo aumento em largura e profundidade do organismo (Siqueira, 1996).

Durante a reposição de nutrientes ou realimentação, a velocidade de ganho de peso é maior nos animais que sofreram restrição alimentar, ocorrendo, conseqüentemente, o ganho compensatório. Vários fatores podem influenciar o ganho compensatório, entre eles, a idade, a severidade e a duração da restrição, sendo possíveis quatro respostas de um animal realimentado após período de restrição alimentar: compensação total, compensação parcial, não compensação ou redução do peso adulto (Ryan, 1990).

Kamalzadeh et al. (1997) verificaram ganho compensatório em cordeiros Swifter (Flemish x Texel) com 14 semanas de idade e peso corporal de $27 \mathrm{~kg}$ submetidos a restrição alimentar qualitativa com forragem de baixa qualidade $(51 \mathrm{~g}$ PB/kg MS), à vontade, em comparação a um grupo controle, recebendo a mesma forragem e suplementação com concentrado (173 g PB/kg MS). Na fase de restrição alimentar, os cordeiros do grupo sem restrição alimentar e com restrição alimentar consumiram $36,9 \mathrm{e} 48,45 \mathrm{~g} / \mathrm{kg}^{0,75} /$ dia de volumoso e tiveram ganho de peso de $8,4 \mathrm{e}-0,72 \mathrm{~g} / \mathrm{kg}^{0,75} / \mathrm{dia}$, respectivamente. Durante a fase de ganho compensatório, os cordeiros que receberam alimentação restrita passaram a receber $35 \mathrm{~g} / \mathrm{kg}^{0,75} / \mathrm{dia}$ de concentrado suplementar, o que determinou maior consumo de $\mathrm{MO}\left(58,38\right.$ vs $65,75 \mathrm{~g} / \mathrm{kg}^{0,75} /$ dia) e mais alto ganho de peso (6,21 vs $\left.10,72 \mathrm{~g} / \mathrm{kg}^{0,75} / \mathrm{dia}\right)$. Portanto, durante a realimentação, os animais submetidos à restrição alimentar foram mais eficientes na conversão alimentar, com menor consumo total de alimento, de modo que o atraso no crescimento foi compensado.

Kamalzadeh et al. (1998) estudaram o efeito da duração da restrição alimentar qualitativa sobre o peso corporal e as medidas biométricas de cordeiros distribuídos em três tratamentos (controle, sem restrição alimentar durante todo experimento; restrição alimentar por três meses; e restrição alimentar por 4,5 meses) e verificaram reduções no peso corporal e nas medidas biométricas durante a fase de restrição alimentar. $\mathrm{Na}$ fase de realimentação, o peso corporal e as medidas biométricas dos animais sob restrição por três meses foram totalmente compensados (99\%). Contudo, nos animais sob restrição alimentar por 4,5 meses, o peso corporal e a maioria das medidas corporais (à exceção da altura na cernelha e do comprimento de Ulna - compensação total) foram compensados parcialmente (55\%). Os autores concluíram que restrição alimentar por períodos maiores que três meses não deve ser recomendada, pois seria necessário um longo período para compensar as perdas.

No intuito de avaliar o ganho compensatório de cordeiros Malpura em fase de terminação, Karim et al. (2001) realizaram um estudo com animais em três grupos nutricionais (alto, médio e baixo conteúdo de PB e energia digestível), alimentados por 90 dias pré-terminação e avaliados dos 90 aos 180 dias de idade recebendo dieta com relação volumoso: concentrado de 40:60. Os cordeiros que receberam baixo nível nutricional apresentaram os maiores pesos ao abate e da carcaça, com valores de 23,2 e 10,0; 17,9 e 7,$2 ; 24,2$ e $10,5 \mathrm{~kg}$, respectivamente, para os trata- 
mentos de baixo, médio e alto nível nutricional, comprovando o benefício do ganho compensatório no desempenho de cordeiros em terminação.

Objetivou-se avaliar os efeitos da restrição alimentar e da posterior realimentação sobre as variações de peso, o consumo de matéria seca, a conversão alimentar e as medidas biométricas em cordeiras na fase de recria.

\section{Material e Métodos}

O trabalho foi realizado no Setor de Ovinocultura do Departamento de Zootecnia da Faculdade de Ciências Agrárias e Veterinárias - Unesp, em Jaboticabal, SP. Foram utilizadas 18 cordeiras 7/8 Ile de France 1/8 Ideal com quatro meses de idade e peso corporal médio de $32 \mathrm{~kg}$, alojadas em baias individuais providas de comedouro e bebedouro. A relação volumoso:concentrado da dieta foi de 50:50, formulada para atender às exigências para ganho de $200 \mathrm{~g} / \mathrm{dia}$ segundo o NRC (1985), utilizando-se como volumoso feno de capim-tifton 85 (de baixa qualidade em virtude das condições climáticas desfavoráveis durante a colheita), triturado em partículas de 3 a $5 \mathrm{~cm}$. As composições centesimal e bromatológica da dieta - esta última realizada de acordo com metodologias descritas por Silva \& Queiroz (2002) - podem ser visualizadas na Tabela 1.

O experimento foi dividido em dois períodos, cada um com 60 dias de duração, sendo que, no primeiro período, os animais foram distribuídos em três tratamentos: sem restrição (sem restrição alimentar por todo experimento); restrição 30\% (com restrição alimentar de $30 \%$ em relação ao consumo dos animais do tratamento sem restrição) e restrição $60 \%$ (com restrição alimentar de $60 \%$ em relação ao consumo dos animais do tratamento sem restrição).

Durante o período de restrição alimentar, não houve sobras de alimento nos tratamentos com restrição. No período de realimentação, o alimento foi fornecido à vontade, mantendo-se a relação volumoso:concentrado de acordo com as sobras de feno. $\mathrm{O}$ volumoso foi fornecido uma vez ao dia, às $8 \mathrm{~h}$, e o concentrado, duas vezes ao dia, metade com o volumoso e o restante às $16 \mathrm{~h}$, com ajustes diários para permitir de 10 a $15 \%$ de sobras.

No início do experimento, ao final da restrição e ao final da realimentação, foram registradas as seguintes medidas biométricas (Searle etal., 1989; Yáñezetal., 2004) das cordeiras: altura da cernelha (distância entre a região da cernelha e a extremidade distal do membro anterior) e altura do vazio subesternal (distância entre o solo e o esterno), obtidas com uso de uma régua ovimétrica; profundidade torácica (diferença entre a altura de cernelha e a altura do vazio), comprimento corporal (distância entre a articulação cérvico-torácica e a base da cauda na primeira articulação intercoccígea) e perímetro torácico (perímetro tomando-se como base o esterno e a cernelha), medidos com fita métrica; e largura da garupa (distância entre os trocânteres maiores dos fêmures) e do ombro (distância entre as faces laterais das articulações escápulo-umerais), determinada com o uso de um compasso. A condição corporal foi estimada de acordo com Russel et al. (1969) e Ribeiro et al. (2003) e consistiu na palpação da região dorsal da coluna vertebral, verificando-se a quantidade de gordura e músculo no ângulo formado pelos processos dorsais e transversos, atribuindo-se nota de 1 a $5 \pm 0,5$, em que 1 representa um animal caquético e 5, um animal obeso.

O ganho compensatório foi determinado utilizando-se a fórmula: $\mathrm{C}(\%)=100 \mathrm{x}(\mathrm{A}-\mathrm{B}) / \mathrm{A}$, em que $\mathrm{C}=$ compensação em porcentagem, ou seja, quando $\mathrm{C}=100 \%$, o valor perdido durante a restrição foi recuperado totalmente durante a realimentação; $\mathrm{A}=$ diferença entre a medida dos animais em restrição e a dos animais controle ao final do período de restrição; $\mathrm{B}=$ diferença entre a medida dos animais que sofreram restrição e a dos animais controle ao final do período de realimentação (Wilson \& Osbourn, 1960).

A pesagem do alimento fornecido e das sobras foi feita diariamente para estimativa do consumo de MS e a das cordeiras, semanalmente, pela manhã, após jejum de sólidos de 8 horas, para averiguação da alteração de peso e da conversão alimentar.

O delineamento experimental utilizado foi o inteiramente casualizado, com seis repetições. Os dados foram

Tabela 1 - Composições centesimal e bromatológica da dieta experimental Table 1 - Ingredient and chemical compositions of the experimental diet

\begin{tabular}{|c|c|c|c|c|c|c|c|c|}
\hline & $\begin{array}{c}\text { Dieta }(\%) \\
\text { Diet }(\%)\end{array}$ & $\begin{array}{c}\mathrm{MS}(\%) \\
D M\end{array}$ & $\begin{array}{c}\mathrm{PB}(\%) \\
C P\end{array}$ & $\begin{array}{c}\text { FDN }(\%) \\
N D F\end{array}$ & $\begin{array}{c}\text { FDA }(\%) \\
A D F\end{array}$ & $\begin{array}{c}\mathrm{EE}(\%) \\
E E\end{array}$ & $\begin{array}{c}\text { MM }(\%) \\
\text { Ash }\end{array}$ & $\begin{array}{c}\mathrm{NDT}^{2}(\%) \\
T D N\end{array}$ \\
\hline Concentrado $^{1}$ (Concentrate) & 50,0 & 89,7 & 16,9 & 23,1 & 4,6 & 2,6 & 7,5 & 82,0 \\
\hline Feno Tifton (Tifton hay) & 50,0 & 93,2 & 3,3 & 79,6 & 54,9 & 0,6 & 5,3 & 48,0 \\
\hline Dieta (Diet) & 100,0 & 91,4 & 10,1 & 51,3 & 29,7 & 1,6 & 6,4 & 66,0 \\
\hline
\end{tabular}

${ }^{1} 60 \%$ grão de milho moído, $20 \%$ farelo de soja, $15 \%$ farelo de trigo, $5 \%$ núcleo mineral e vitamínico.

2 Valores estimados de NDT, segundo o NRC (1985).

$160 \%$ ground corn, $20 \%$ soybean meal, $15 \%$ wheat bran, $5 \%$ mineral and vitamin premix.

2 Estimated NDT values (NRC, 1985) 
submetidos ao teste $\mathrm{F}$ de análise de variância e as médias, comparadas pelo teste Tukey, a 5\% de probabilidade (Banzatto \& Kronka, 1992), utilizando-se o seguinte modelo matemático:

$$
\mathrm{Y}_{\mathrm{ij}}=\mu+\mathrm{T}_{\mathrm{i}}+\mathrm{e}_{\mathrm{i}}
$$

em que $Y_{i j}=$ valor observado da variável estudada no indivíduo $\mathrm{j}$ recebendo o tratamento $\mathrm{i} ; \mu=$ média geral; $\mathrm{T}_{\mathrm{i}}=$ efeito do tratamento $\mathrm{i} ; \mathrm{e}_{\mathrm{ij}}=$ erro aleatório associado a cada observação $Y_{\mathrm{ij}}$.

\section{Resultados e Discussão}

As cordeiras do tratamento sem restrição apresentaram ganhos de $113,77 \mathrm{~g} / \mathrm{dia}$, com aumento de $18 \%$ do peso corporal final em relação ao peso inicial. As cordeiras do grupo restrição $30 \%$ tiveram ganho de $4,43 \mathrm{~g} /$ dia, enquanto as do grupo restrição $60 \%$ perderam peso $(80,33 \mathrm{~g} / \mathrm{dia})$, resultando em diminuição de $15 \%$ do peso corporal inicial ao final do período de restrição alimentar. Como demonstrado na Tabela 2, no período de restrição alimentar, não houve valores de conversão alimentar para os grupos restrição $30 \%$ e restrição $60 \%$, pois os valores tiveram grande variação em decorrência da alteração de peso, que, aliada ao baixo consumo, enseja valores absurdos desse índice.

Ao final do experimento, o peso corporal foi compensado parcialmente em 91,5 e $49,7 \%$, de acordo com o índice compensatório de Wilson \& Osbourn (1960), nas cordeiras dos grupos restrição $30 \%$ e restrição $60 \%$, que, ao início do período de realimentação, pesaram 32,3 e $28,0 \mathrm{~kg}$, respecti- vamente. A velocidade de crescimento dos animais sem restrição $(y=0,92 x+37,33)$ foi inferior à daqueles submetidos à restrição $60 \%(\mathrm{y}=1,22 \mathrm{x}+27,39)$, mas, em ambos os grupos, foi menor que a do grupo restrição $30 \%(y=1,39 x$ $+32,39$ ). Representando o ganho compensatório dos grupos restrição $30 \%$ e restrição $60 \%$ durante o período de realimentação, os coeficientes angulares das equações das curvas de alterações dos pesos corporais foram superiores aos obtidos no grupo sem restrição (Figura 1).

O consumo de MS ( $\left.\mathrm{g} / \mathrm{kg}^{0,75} / \mathrm{dia}\right)$ pelos animais do grupo sem restrição durante o período de realimentação apresentou aumento constante, porém sempre inferior ao das cordeiras do grupo restrição $30 \%$, que apresentaram acréscimo do consumo até a 4 a semana de realimentação, quando o consumo começou a decrescer até a $8^{\mathrm{a}}$ semana, se assemelhando à ingestão das cordeiras sem restrição. As cordeiras do grupo restrição $60 \%$ no início da realimentação tiveram aumento na ingestão até a $3 \underline{\text { a }}$ semana, porém esse aumento foi sempre inferior ao das cordeiras dos demais grupos (Figura 2). A restrição alimentar moderada (restrição $30 \%$ ) possibilitou elevação do consumo, ao passo que a restrição severa (restrição 60\%) não elevou o consumo e ainda prejudicou a ingestão.

No período de realimentação, esperava-se maior consumo no grupo restrição $60 \%$, no entanto, ocorreu o inverso, provavelmente em virtude do alto grau de restrição alimentar ao qual foram submetidas no período de restrição, não propiciando o ganho compensatório total e evidenciando a severidade da restrição sobre a redução no

Tabela 2 - Peso corporal inicial, peso corporal final, consumo de MS, alteração de peso e conversão alimentar de cordeiras em crescimento no período de restrição alimentar

Table 2 - Initial body weight, final body weight, DM intake, body weight change and feed conversion of growing female lambs during the feed restriction period

\begin{tabular}{|c|c|c|c|c|c|}
\hline \multirow[b]{2}{*}{$\begin{array}{l}\text { Período } \\
\text { Period }\end{array}$} & \multirow[b]{2}{*}{$\begin{array}{l}\text { Parâmetro } \\
\text { Parameter }\end{array}$} & \multicolumn{3}{|c|}{$\begin{array}{c}\text { Tratamento }^{1} \\
\text { Treatment }\end{array}$} & \multirow[b]{2}{*}{$\mathrm{CV}(\%)^{3}$} \\
\hline & & $\begin{array}{c}\text { Sem restrição } \\
\text { Without restriction }\end{array}$ & $\begin{array}{l}\text { Restrição } 30 \% \\
\text { Restriction } \\
30 \%\end{array}$ & $\begin{array}{l}\text { Restrição } 60 \% \\
\text { Restriction } 60 \%\end{array}$ & \\
\hline & $\begin{array}{l}\text { PI (kg) } \\
I W(k g)\end{array}$ & $32,08 \mathrm{a}$ & $33,02 \mathrm{a}$ & $32,82 \mathrm{a}$ & 5,24 \\
\hline & $\begin{array}{l}\mathrm{PF}(\mathrm{kg}) \\
F W(k g)\end{array}$ & $38,38 \mathrm{a}$ & $33,28 b$ & $28,00 \mathrm{c}$ & 6,82 \\
\hline Restrição & $\begin{array}{l}\text { AP (g/dia) } \\
W A(g / d a y)\end{array}$ & $113,77 \mathrm{a}$ & $4,43 b$ & $-80,33 c$ & 401,19 \\
\hline \multirow[t]{4}{*}{ Restriction } & $\begin{array}{l}\text { CMS (kg/dia) } \\
D M I \text { (kg/day) }\end{array}$ & $1,05 \mathrm{a}$ & $0,73 b$ & $0,43 \mathrm{c}$ & 15,57 \\
\hline & $\begin{array}{l}\text { CMS (\%PV) } \\
D M I(\% B W)\end{array}$ & $2,93 \mathrm{a}$ & $2,19 b$ & $1,53 \mathrm{c}$ & 8,37 \\
\hline & $\begin{array}{l}\text { CMS }\left(\mathrm{g} / \mathrm{kg}^{0,75} / \mathrm{dia}\right) \\
\text { DMI }\left(\mathrm{g} / \mathrm{kg}^{0,75} / \text { day }\right)\end{array}$ & $71,43 a$ & $52,50 \mathrm{~b}$ & $35,17 \mathrm{c}$ & 8,71 \\
\hline & $\begin{array}{l}\mathrm{CA} \\
F C\end{array}$ & 11,12 & - & - & - \\
\hline
\end{tabular}

\footnotetext{
${ }^{1}$ Médias seguidas de letras diferentes na linha diferem $(P<0,05)$ pelo teste Tukey.
}

1 Means followed by different letters in a row differ $(P<0.05)$ by Tukey test. 


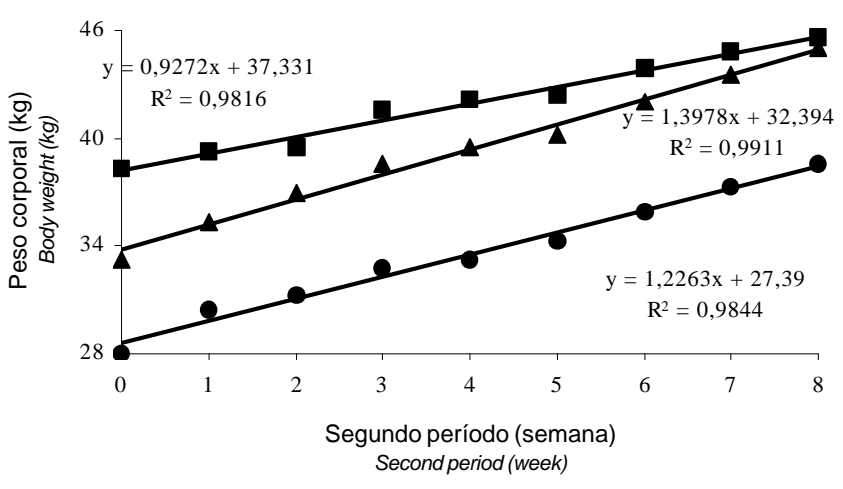

sem restrição; $\mathbf{\Lambda}$ restrição 30\%; — restrição 60\%

- without restriction; $\mathbf{A}$ restriction 30\%; - restriction 60\%

Figura 1 - Equações e correlações das curvas de alterações dos pesos corporais de cordeiras durante o período de realimentação.

Figure 1 - Equations and correlation curves for body weigh change of growing female lambs during the second period.

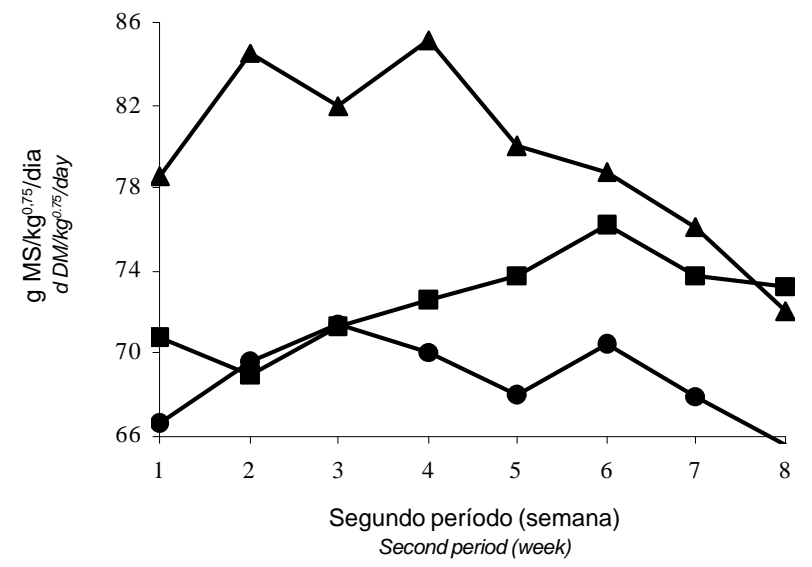

sem restrição; $\boldsymbol{\Delta}$ restrição 30\%; restrição 60\%

without restriction; $\mathbf{\Delta}$ restriction $30 \%$; restriction $60 \%$

Figura 2 - Consumo de MS em g MS/kg $0,75 /$ dia pelas cordeiras durante o período de realimentação à vontade.

Figure 2 - DM intake in $\mathrm{D} D M / \mathrm{kg}^{0,75} /$ day of growing female lambs during the second period.

consumo (Ryan, 1990). Não houve diferença $(\mathrm{P}<0,05)$ no consumo de MS, expresso em $\mathrm{g} / \mathrm{kg}^{0,75} / \mathrm{dia}$ e $\% \mathrm{PV} / \mathrm{dia}$. Sabe-se que animais pesados consomem mais, em $\mathrm{kg} / \mathrm{dia}$, que os leves. As cordeiras do tratamento restrição $30 \%$ consumiram mais que as do grupo sem restrição, enquanto as do grupo restrição $60 \%$ consumiram menos $(\mathrm{P}>0,05)$ que aquelas sem restrição (Tabela 3 ).

Constatou-se melhor aproveitamento do alimento no período de realimentação pelas cordeiras submetidas à restrição alimentar. No entanto, o desempenho das cordeiras do grupo restrição $60 \%$ foi prejudicado pelo baixo consumo. A conversão alimentar não diferiu $(\mathrm{P}>0,05)$ entre os grupos restrição $30 \%$ e restrição $60 \%$, que apresentaram valores de 6,28 e 5,66, respectivamente (Tabela 3 ), melhores $(\mathrm{P}<0,05)$ que a obtida no grupo sem restrição $(10,09)$.

A conversão alimentar foi expressivamente melhor durante o segundo período nos animais previamente subnutridos, indicando a possibilidade de manipular o consumo do cordeiro por um período visando diminuir a relação entre o ganho de peso e o consumo de MS do período seguinte, obtendo-se um consumo mais econômico e sem prejuízos no peso corporal. Na Figura 3, é possível observar o comportamento quadrático positivo e quadrático negativo das curvas de conversão alimentar durante o período de realimentação nas cordeiras sem restrição e com restrição, respectivamente (Figura 3).

Drouillard et al. (1991) submeteram cordeiros a restrição de proteína ou energia, em comparação á ausência de restrição nutricional, e reportaram que a eficiência alimentar, do início da realimentação até a $2^{\mathrm{a}}$ semana, dos animais submetidos a restrição de proteína foi melhor que naqueles que sofreram restrição energética.

Como pode ser visualizado na Figura 4, o ganho compensatório foi mais evidente nas cordeiras submetidas a restrição $30 \%$. A velocidade de ganho de peso ao final do segundo período naquelas sem restrição foi menor (116,2 g/dia) que nas do grupo restrição 30\% (196,24 g/dia), mas essa característica, em ambos os grupos, não diferiu (P>0,05) do grupo restrição 60\% (178,03 g/dia). Kabbali et al. (1992), estudando o ganho compensatório de cordeiros

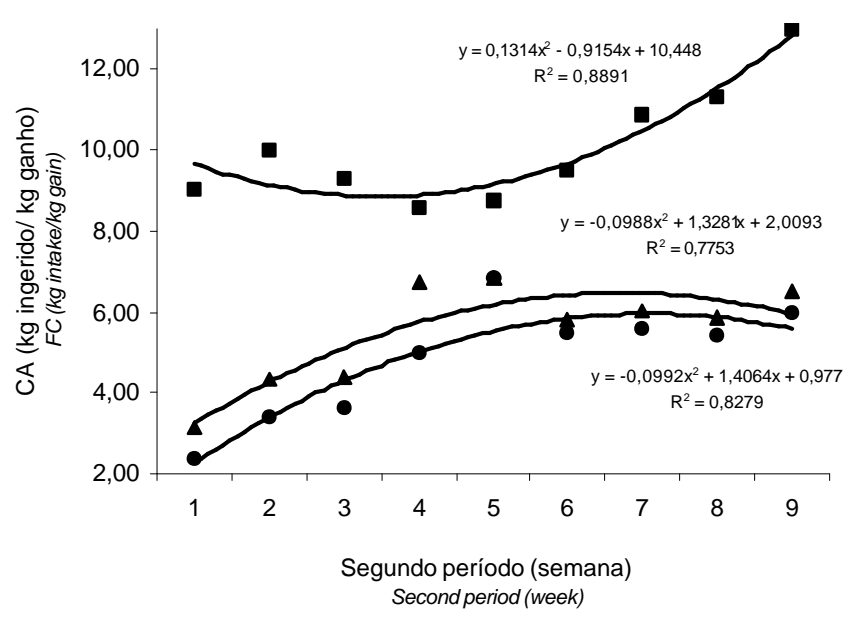

sem restrição; restrição 30\%; restrição 60\%

without restriction; $\mathbf{\Lambda}$ restriction 30\%; restriction $60 \% \%$

Figura 3 - Conversão alimentar das cordeiras durante o período de realimentação.

Figure 3 - Feed conversion of growing female lambs during the second period. 
Tabela 3 - Peso corporal inicial, peso corporal final, consumo de MS, alteração de peso e conversão alimentar de cordeiras em crescimento no período de realimentação

Table 3 - Initial body weight, final body weight, dry matter intake, body weight change and feed conversion of growing female lambs during the second period

\begin{tabular}{|c|c|c|c|c|c|}
\hline \multirow[b]{2}{*}{$\begin{array}{l}\text { Período } \\
\text { Period }\end{array}$} & \multirow[b]{2}{*}{$\begin{array}{l}\text { Parâmetro } \\
\text { Parameter }\end{array}$} & \multicolumn{3}{|c|}{$\begin{array}{c}\text { Tratamento } \\
\text { Treatment }\end{array}$} & \multirow[b]{2}{*}{$\mathrm{CV}(\%)$} \\
\hline & & $\begin{array}{c}\text { Sem restrição } \\
\text { Without restriction }\end{array}$ & $\begin{array}{l}\text { Restrição } 30 \% \\
\text { Restriction } 30 \%\end{array}$ & $\begin{array}{l}\text { Restrição } 60 \% \\
\text { Restriction } 60 \%\end{array}$ & \\
\hline \multirow{9}{*}{$\begin{array}{l}\text { Realimentação } \\
\text { Refeeding }\end{array}$} & $\begin{array}{l}\text { PI }(\mathrm{kg}) \\
I W(k g)\end{array}$ & $38,08 \mathrm{a}$ & $33,23 b$ & $28,00 \mathrm{c}$ & 6,81 \\
\hline & $\begin{array}{l}\mathrm{PF}(\mathrm{kg}) \\
F W(k g)\end{array}$ & $45,03 \mathrm{a}$ & $44,62 \mathrm{a}$ & $39,96 \mathrm{a}$ & 8,74 \\
\hline & $\begin{array}{l}\text { AP }(\text { g/dia }) \\
W A(g / d a y)\end{array}$ & $116,20 \mathrm{~b}$ & $196,24 \mathrm{a}$ & $178,03 \mathrm{ab}$ & 28,35 \\
\hline & CMS (kg/dia) & $1,16 \mathrm{ab}$ & $1,23 \mathrm{a}$ & $1,02 \mathrm{~b}$ & 18,69 \\
\hline & $D M I$ (kg/day) & & & & \\
\hline & CMS (\%PV) & $2,62 \mathrm{a}$ & $2,73 \mathrm{a}$ & $2,42 \mathrm{a}$ & 10,17 \\
\hline & $\begin{array}{l}D M I(\% B W) \\
\text { CMS }\left(\mathrm{g} / \mathrm{kg}^{0,75} / \mathrm{dia}\right) \\
D M I\left(g / \mathrm{kg}^{0.75} / \text { day }\right)\end{array}$ & $66,98 \mathrm{a}$ & $70,80 \mathrm{a}$ & $60,62 \mathrm{a}$ & 11,62 \\
\hline & $\mathrm{CA}$ & $10,09 \mathrm{a}$ & $6,28 b$ & $5,66 \mathrm{~b}$ & 24,36 \\
\hline & $F C$ & & & & \\
\hline
\end{tabular}

${ }^{1}$ Médias seguidas de letras diferentes na linha diferem $(P<0,05)$ pelo teste Tukey.

${ }^{1}$ Means followed by different letters in a row differ $(P<0.05)$ by Tukey test.

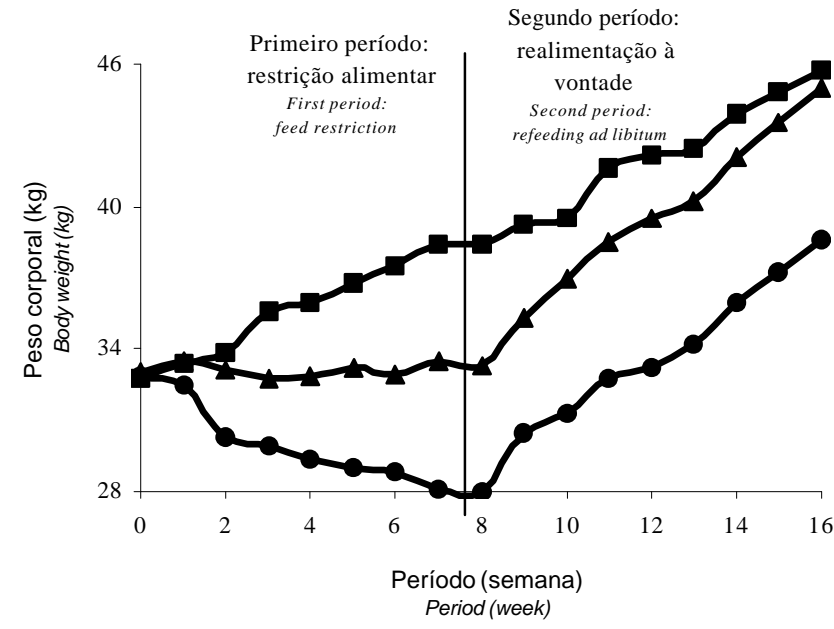

sem restrição; $\mathbf{\Lambda}$ restrição $30 \%$; restrição $60 \%$

without restriction; $\mathbf{\Lambda}$ restriction 30\%; restriction $60 \%$

Figura 4 - Alteração no peso corporal durante os períodos de restrição alimentar e de realimentação (ganho compensatório) em cordeiras na fase de recria.

Figure 4 - Body weight change during the first (feed restriction) and second (compensatory growth) periods in growing female lambs.

dos 17 aos $21 \mathrm{~kg}$ submetidos à restrição nutricional de energia, constataram maior diferença no ganho de peso ( $355 \mathrm{~g} / \mathrm{dia})$ entre animais que passaram por restrição, comparados aos que não foram submetidos a restrição alimentar (208 g/dia).

Se o ritmo de crescimento fosse mantido e o período de realimentação fosse aumentado, as cordeiras do grupo restrição $60 \%$ provavelmente atingiriam o peso corporal daquelas sem restrição e aquelas do grupo restrição $30 \%$ apresentariam peso corporal superior ao das sem restrição. Segundo Ryan (1990), a severidade da restrição afeta o tempo de permanência em ganho compensatório e o grau de compensação.

Ao final do experimento, animais do grupo restrição $60 \%$ deixaram de ganhar $5 \mathrm{~kg}$ em relação àqueles sem restrição, no entanto, a economia de alimento no período total foi de $35,6 \%$. As cordeiras sem restrição consumiram $115,86 \mathrm{~kg}$, as do grupo restrição $30 \% 106,05 \mathrm{~kg}$ e as do grupo restrição $60 \% 74,55 \mathrm{~kg}$, o que representa uma diferença de $8,5 \%$ para as cordeiras do grupo restrição $30 \%$ e de $35,6 \%$ para as do grupo restrição $60 \%$ em relação àquelas sem restrição (Tabela 4), o que está de acordo com os relatos de Kamalzadeh et al. (1997), que obtiveram menor consumo total de alimento em ovinos submetidos a restrição alimentar qualitativa.

$\mathrm{Na}$ Tabela 5 constam as medidas biométricas das cordeiras do grupo sem restrição, restrição $30 \%$ e restrição $60 \%$ ao início e final do primeiro período (restrição alimentar) e ao final do segundo período (realimentação). Ao início da fase de restrição alimentar, todas as cordeiras apresentaram medidas biométricas semelhantes $(\mathrm{P}>0,05)$, mas, ao final da restrição alimentar, houve diferença $(\mathrm{P}<0,05)$ entre os animais do grupo restrição $60 \% \mathrm{em}$ relação aos de restrição $30 \%$ e sem restrição. Houve diminuição de $16 \%$ na largura do ombro, $21 \%$ na largura da garupa, $6,9 \%$ no perímetro torácico e $39 \%$ na condição corporal das cordeiras do grupo restrição $60 \%$ em relação à medida inicial. Todavia, não houve diferença entre os 
Tabela 4 - Consumo de MS das cordeiras nos períodos de restrição alimentar, de realimentação e durante todo período experimental Table 4 - DM intake of growing female lambs during the first (feed restriction) and second (compensatory growth) periods and during all experimental period

\begin{tabular}{lccc}
\hline $\begin{array}{l}\text { Período } \\
\text { Period }\end{array}$ & $\begin{array}{c}\text { Sem restrição (kg) } \\
\text { Without restriction }\end{array}$ & $\begin{array}{c}\text { Restrição 30\% (kg) } \\
\text { Restriction 30\% }\end{array}$ & $\begin{array}{c}\text { Restrição 60\% (kg) } \\
\text { Restriction 60\% }\end{array}$ \\
\hline Restrição alimentar (60 dias) (Feed restriction, 60 days) & 55,49 & 38,90 & 22,91 \\
Realimentação (60 dias) (Refeeding, 60 days) & 60,37 & 67,14 & 51,63 \\
Total (120 dias) (All, 120 days) & 115,86 & 106,05 & 74,55 \\
Economia de alimento $(\%)^{1}$ (Feed saving, \%) & 0,00 & 8,50 & 35,60 \\
\hline
\end{tabular}

${ }^{1}$ Consumo de MS do período total em relação ao consumo das cordeiras sem restrição.

1 DM intake during the total experimental period in relation to the intake with no feed restriction.

Tabela 5 - Média e desvio-padrão de medidas biométricas das cordeiras ao início e final da restrição alimentar e ao final da realimentação Table 5 - Means and standard deviation of biometrical measurements of growing female lambs at the beginning and end of the feed restriction period and at the end of the second period

\begin{tabular}{lccc}
\hline $\begin{array}{l}\text { Tratamento } \\
\text { Treatment }\end{array}$ & Início restrição alimentar & Fim restrição alimentar & Fim realimentação \\
Beginning feed restriction & End feed restriction & refeeding \\
\hline
\end{tabular}

Comprimento corporal, $\mathrm{cm}$

Body length, $\mathrm{cm}$

Sem restrição

Restrição $30 \%$

Restrição $60 \%$

Perímetro torácico, $\mathrm{cm}$

Chest girth, $\mathrm{cm}$

Sem restrição

Restrição $30 \%$

Restrição 60\%

Altura na cernelha, cm

Withers heights, $\mathrm{cm}$

Sem restrição

Restrição 30\%

Restrição 60\%

Altura do vazio subesternal, $\mathrm{cm}$

Withers heights, $\mathrm{cm}$

Sem restrição

Restrição $30 \%$

Restrição $60 \%$

Profundidade torácica, $\mathrm{cm}$

Chest depth, $\mathrm{cm}$

Sem restrição

Restrição 30\%

Restrição $60 \%$

Largura de ombro, $\mathrm{cm}$

Shoulder width, $\mathrm{cm}$

Sem restrição

Restrição 30\%

Restrição 60\%

Largura de garupa, cm

Chest depth, $\mathrm{cm}$

Sem restrição

Restrição 30\%

Restrição $60 \%$

Condição corporal

Body condition

\begin{tabular}{ll} 
T 1 & $3,4 \pm 0,82 \mathrm{a}$ \\
T 2 & $4,0 \pm 0,45 \mathrm{a}$ \\
T 3 & $3,7 \pm 0,26 \mathrm{a}$ \\
\hline
\end{tabular}

$25,52 \pm 0,99$ a
$57,4 \pm 3,91 \mathrm{a}$

$57,9 \pm 1,58 \mathrm{a}$

$58,8 \pm 2,92 \mathrm{a}$

$65,4 \pm 3,36 \mathrm{a}$

$64,2 \pm 3,63 \mathrm{a}$

$62,8 \pm 2,58$ a

$75,6 \pm 2,30 \mathrm{a}$

$78,1 \pm 2,18 \mathrm{a}$

$76,4 \pm 3,98$ a

$54,5 \pm 1,22 \mathrm{a}$

$53,8 \pm 1,92 \mathrm{a}$

$56,2 \pm 1,25 \mathrm{a}$

$30,3 \pm 3,07 \mathrm{a}$

$30,4 \pm 1,50$ a

$31,0 \pm 0,26 \mathrm{a}$

$24,2 \pm 2,65 \mathrm{a}$

$23,3 \pm 2,49$ a

$25,1 \pm 1,08 \mathrm{a}$

$23,1 \pm 1,18 \mathrm{a}$

$23,3 \pm 1,43 a$

$22,8 \pm 2,28$ a

$23,68 \pm 2,07$ a

$24,46 \pm 0,91$ a

$3,7 \pm 0,26 \mathrm{a}$
$76,4 \pm 4,56 \mathrm{a}^{1}$

$76,4 \pm 2,70 \mathrm{a}$

$71,2 \pm 2,38 \mathrm{~b}$

$58,6 \pm 2,50 \mathrm{a}$

$60,4 \pm 0,54 \mathrm{a}$

$58,8 \pm 0,44 \mathrm{a}$

$31,0 \pm 2,44 \mathrm{a}$

$33,4 \pm 1,51$ a

$32,6 \pm 2,07 \mathrm{a}$

$27,6 \pm 2,96 \mathrm{a}$

$27,0 \pm 1,58 \mathrm{a}$

$26,2 \pm 2,38 b$

$23,8 \pm 1,44 \mathrm{a}$

$22,0 \pm 0,79$ a

$19,0 \pm 1,00 \mathrm{~b}$

$24,2 \pm 1,52 \mathrm{a}$

$22,5 \pm 1,65 \mathrm{ab}$

$20,4 \pm 2,16 b$

$24,4 \pm 1,38 \mathrm{a}$

$23,0 \pm 1,41 \mathrm{a}$

$19,3 \pm 2,10 \mathrm{~b}$

$25,2 \pm 1,15 \mathrm{a}$

$24,9 \pm 0,41 \mathrm{a}$

$21,6 \pm 2,27 \mathrm{~b}$

${ }_{1}^{1}$ Médias seguidas de letras diferentes na linha diferem $(P<0,05)$ pelo teste Tukey.

1 Means followed by different letters in a row differ $(P<0.05)$ by Tukey test.

$\begin{array}{ll}3,8 \pm 0,75 \mathrm{a} & 3,8 \pm 0,83 \mathrm{a} \\ 3,2 \pm 0,27 \mathrm{a} & 3,8 \pm 0,27 \mathrm{a} \\ 2,3 \pm 0,27 \mathrm{~b} & 3,5 \pm 0,35 \mathrm{a}\end{array}$


animais sem restrição e os do grupo restrição $30 \%$ para nenhum dos parâmetros mensurados ao final dos períodos de restrição e de realimentação.

As dimensões corporais reagiram diferentemente à restrição alimentar e, durante a realimentação, em geral as medidas de altura na cernelha e comprimento corporal foram menos afetadas que a largura e o perímetro torácico. A largura de ombro das cordeiras do grupo restrição $60 \%$ foi recuperada $(\mathrm{P}>0,05)$ com a realimentação, o mesmo não ocorrendo com as outras medidas corporais ao final desse período. Kamalzadeh et al. (1998) reportaram que as medidas biométricas menos influenciadas pela restrição alimentar são também as de menor compensação na realimentação.

A severidade da restrição $60 \%$ foi tão grande que ascordeiras não recuperaram o tamanho corporal nas oito semanas de realimentação, ao passo que aquelas do grupo restrição 30\%, apesar da redução nodesenvolvimento normal, não diferiram $(\mathrm{P}>0,05)$ daquelas sem restrição ao final da primeira fase, acompanhando as cordeiras sem restrição até o final da realimentação.
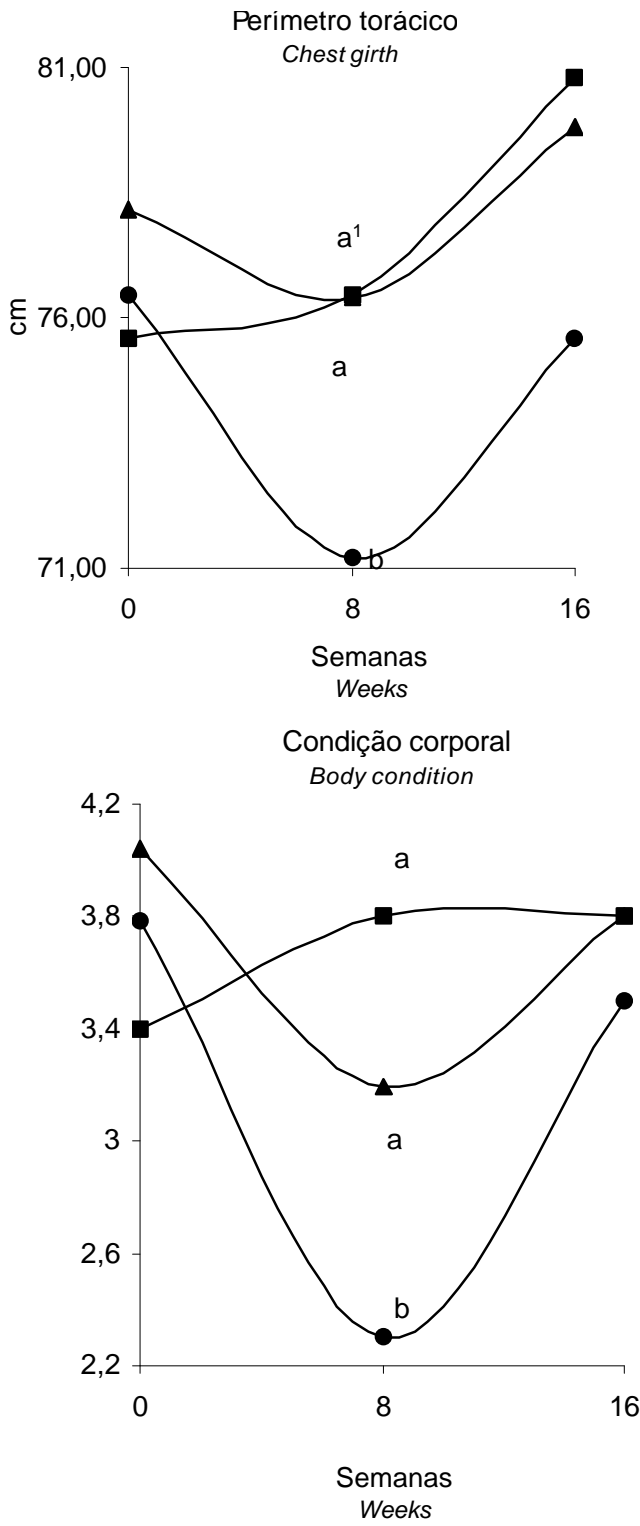
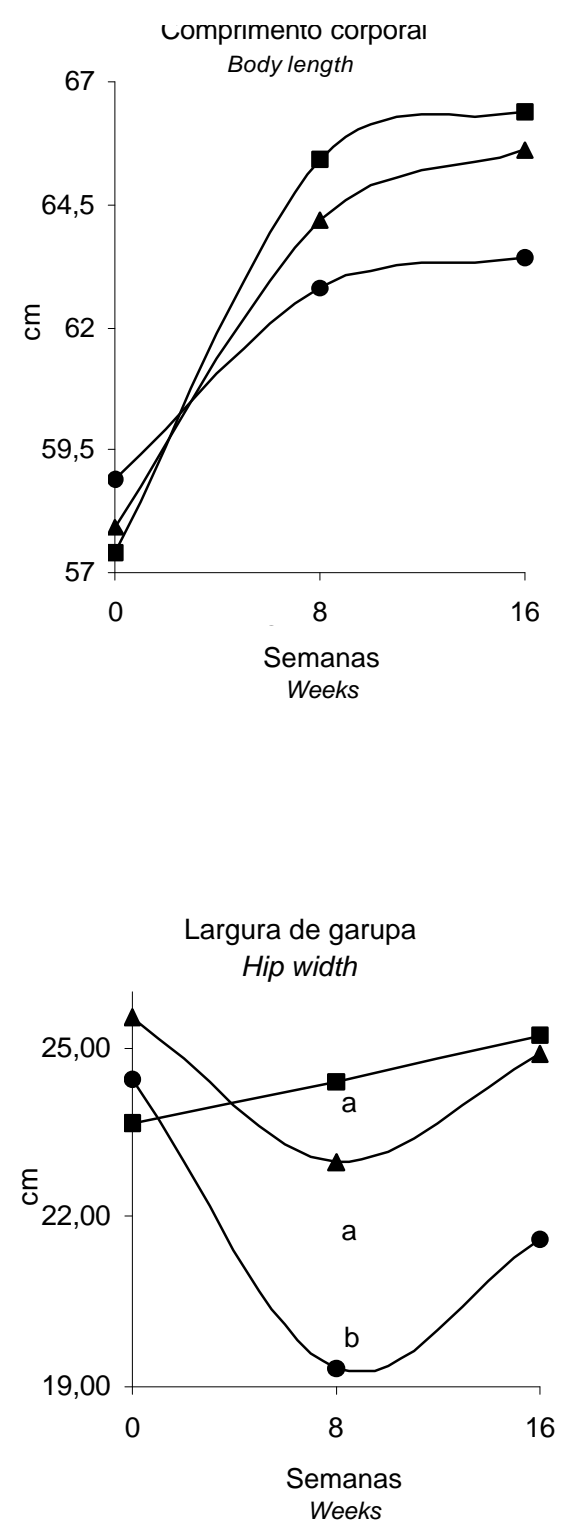

\footnotetext{
1 Letras diferentes nos gráficos indicam diferença $(\mathrm{P}<0,05)$ pelo teste Tukey.

1 Different letters in the graphs indicate difference $(P<0.05)$ by Tukey test.

a sem restrição; $\Lambda$ restrição 30\%; • restrição $60 \%$

- without restriction; $\mathbf{\Lambda}$ restriction $30 \%$

restriction $60 \%$
}

Figura 5 - Alterações biométricas de cordeiras nos períodos de restrição e realimentação.

Figure 5 - Biometrical measurements change in growing female lambs during the first (feed restriction) and second (compensatory growth) periods. 
Foram observadas reduções das dimensões corporais dos animais em restrição alimentar, sobretudo nas cordeiras restrição $60 \%$, que diferiram $(\mathrm{P}<0,05)$ daquelas dos grupos restrição $30 \%$ e sem restrição. Ao final da realimentação, houve recuperação das medidas corporais, mas a defasagem corporal das cordeiras restrição $60 \%$ não se equiparou à daquelas sem restrição. A restrição alimentar de $60 \%$ reduziu o crescimento normal das cordeiras, observando-se diferença $(\mathrm{P}<0,05)$ quanto à largura de garupa, ao perímetro torácico e à condição corporal entre as cordeiras dos grupos restrição $30 \%$ e sem restrição (Figura 5).

\section{Conclusões}

A restrição alimentar de $30 \%$ pode ser adotada como prática de manejo nutricional para períodos de escassez de alimento ou para ovinos que serão submetidos a confinamento como forma de melhorar o ganho de peso e reduzir o tempo de confinamento, o consumo total de alimento e, conseqüentemente, os custos com alimentação. As restrições mais severas, como a de $60 \%$, não são recomendadas, pois podem prejudicar o desempenho e as medidas biométricas de cordeiras.

\section{Literatura Citada}

ANUAlPEC. Anuário da pecuária brasileira. São Paulo: FNP Consultoria \& Agroinformativo, 2004. 376p.

BANZATTO, D.A.; KRONKA, S.N. Experimentação agrícola: estatística experimental. 2.ed. Jaboticabal: FUNEP, 1992. 247p.

DROUILLARD, J.S.; KLOPFENSTEIN, T.J.; BRITTON, R.A. et al. Growth, body composition, and visceral organ mass and metabolism in lambs during and after metabolizable protein or net energy restrictions. Journal of Animal Science, v.69, n.8, p.3357-3375, 1991 .

GERASEEV, L.C.; PEREZ, J.R.O.; CARVALHO, P.A. et al. Efeitos das restrições pré e pós-natal sobre o crescimento e desempenho de cordeiros Santa Inês do desmame ao abate. Revista Brasileira de Zootecnia, v.35, n.1, p.237-244, 2006.

KABBALI, A.; JOHNSON, W.L.; JOHNSON, D.W. et al. Effects of undernutrition and refeeding on weights of body parts and chemical components of growing Moroccan lambs. Journal of Animal Science, v.70, n.9, p.2859-2865, 1992.
KAMALZADEH, A.; van BRUCHEM, J.; KOOPS, W.J. et al. Feed quality restriction and compensatory growth in growing sheep: feed intake, digestion, nitrogen balance and modeling changes in feed efficiency. Livestock Production Science, v.52, n.3, p.209-217, 1997.

KAMALZADEH, A.; KOOPS, W.J.; van BRUCHEM, J. et al. Effect of duration of feed quality restriction on body dimensions in lambs. Journal of Animal Science, v.76, n.3, p.735-742, 1998.

KARIM, S.A.; SANTRA, A.; SEM, A.R. et al. Carcass characteristics of intensively fed finisher lambs maintained on varying plane of nutrition in pre-weaning phase. Indian Journal of Animal Sciences, v.1, n.10, p.955-958, 2001.

NATIONAL RESEARCH COUNCIL - NRC. Nutrient requirements of sheep. 6.ed. Washington, D.C.: National Academy Press, 1985. 99p.

RIBEIRO, L.A.O.; FONTANA, C.S.; WALD, V.B. et al. Relação entre a condição corporal e a idade das ovelhas no encarneiramento com a prenhez. Ciência Rural, v.33, n.2, p.357-361, 2003.

RUSSEL, A.J.F.; DONEY, J.M.; GUNN, R.G. Subjective assessment of body fat in live sheep. Journal of Agricultural Science, v.72, n.3, p.451-454, 1969.

RYAN, W.J. Compensatory growth in cattle and sheep. Nutrition Abstracts Reviews, v.60, n.4, p.653-664, 1990.

SEARLE, T.W.; GRAHAM, McC.; DONNELLY, J.B. Change of skeletal dimensions during growth in sheep: the effect of nutrition. Journal of Agricultural Science, v.112, n.3, p.321-327, 1989.

SILVA, D.J.; QUEIROZ, A.C. Análise de alimentos: métodos químicos e biológicos. 3.ed. Viçosa, MG: Editora UFV, 2002. $235 \mathrm{p}$.

SIQUEIRA, E.R. Recria e terminação de cordeiros em confinamento. In: SILVA SOBRINHO, A.G.; BATISTA, A.M.V.; SIQUEIRA, E.R. et al. (Eds.) Nutrição de ovinos. Jaboticabal: FUNEP, 1996. p. 175-212.

SUSIN, I. Exigências nutricionais de ovinos e estratégias de alimentação. In: SILVA SOBRINHO, A.G.; BATISTA, A.M.V.; SIQUEIRA, E.R. et al. (Eds.) Nutrição de ovinos. Jaboticabal: FUNEP, 1996. p.119-141.

WILSON, P.N.; OSBOURNE, D.F. Compensatory growth after undernutrition in mammals and birds. Biological Reviews, v.35, n.2, p.324-363, 1960.

YÁÑEZ, E.A.; RESENDE, K.T.; FERREIRA, A.C.D. et al. Utilização de medidas biométricas para predizer características da carcaça de cabritos Saanen. Revista Brasileira de Zootecnia, v.33, n.6, p.1564-157, 2004 